\title{
Mapping our profession's future
}

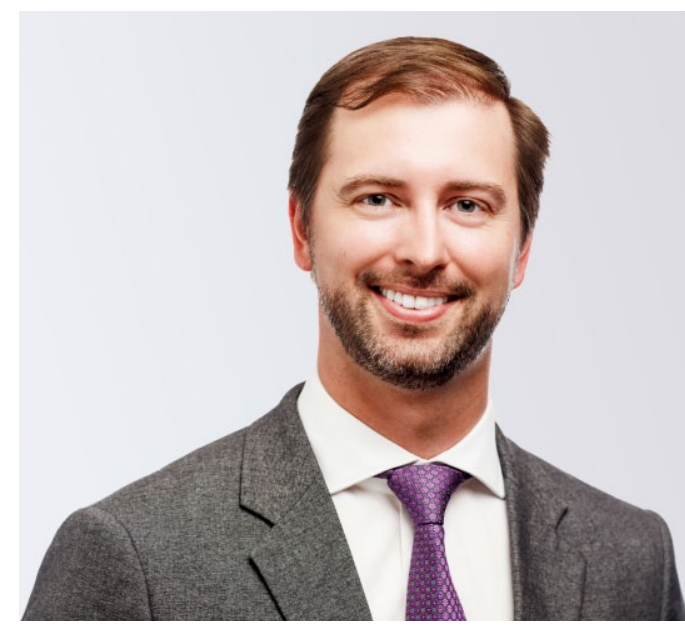

Advancing the profession. Delivering a unified scope. Leading pharmacy practice research.

It has been almost 4 years since $\mathrm{CPhA}$ and the provincial pharmacy associations partnered together to better serve pharmacists. In a short period of time, we have made great strides in moving our profession forward. Although we have come far, there is much work to be done in preparing our practitioners for a rapidly changing future.

In 2017, CPhA launched a brand new strategic plan-a roadmap for success in an ever-changing health care system. Pharmacists want to be part of a new health care solution, and we are more than willing to practise to our fullest potential. We have seen great examples of this practice in action in Alberta, under the leadership of the Alberta College of Pharmacists and the Alberta Pharmacists' Association, resulting in ground-breaking research from a clinical and economic perspective. It's time we take all these innovative and novel examples, from coast to coast, and define a new, long overdue vision for our profession.

This vision is the most important pillar of $\mathrm{CPhA}$ 's new strategic plan. It encompasses an appropriate scope of practice, our patient's needs, a complex health care system, and new, potentially disruptive technology. It needs to be focused on advancing our profession to its fullest potential. And finally, it needs to be a vision that conducts world-leading pharmacy practice research, demonstrating the clinical and economic value of our pharmacist interventions.

$\mathrm{CPhA}$ has tasked our Professional Practice Working Group, led by Iris Krawchenko, with the development of this unified vision. It is critical that pharmacists nationwide put our fragmented scope of practice behind us and work together to fully realize the potential of our profession.

Cheers, Alistair Bursey Chair, Canadian Pharmacists Association Pharmacist | Fredericton, NB 